\title{
L-carnitine in critically ill patients-a case series study
}

\author{
Takehiko Oami ${ }^{1}$, Taku Oshima ${ }^{1 *}$, Noriyuki Hattori', Ayako Teratani', Saori Honda², Toshihiko Yoshida ${ }^{2}$ \\ and Shigeto Oda ${ }^{1}$
}

\begin{abstract}
Background: L-carnitine is essential for lipid metabolism, and lack of L-carnitine intake and loss by treatments lead to carnitine depletion causing muscle weakness, anemia, and immune dysfunction. Carnitine depletion occurs in critically ill patients after long treatments, but its epidemiology or the risk factors remain unclear. This study aims to investigate the prevalence and risk factors of L-carnitine depletion in critically ill patients.

Methods: Sixty-four patients were enrolled for the study. Total and free L-carnitine concentrations (t- and f-Carnitine) were measured at ICU admission and every 7 days afterward during hospitalization. Acylcarnitine- to f-Carnitine ratio (A/F Carnitine ratio) was analyzed in a subgroup of patients treated with continuous renal replacement therapy (CRRT). Acylcarnitine concentration was calculated as the difference between t- and f-Carnitine concentrations.
\end{abstract}

Results: Carnitine deficiency (f-Carnitine $<36 \mathrm{nmol} / \mathrm{mL}$ ) was observed in 15 (23.4\%) patients at ICU admission. Low body mass index (BMI < 19.5) was associated with a subsequent reduction of L-carnitine during the ICU stay (AUC $=0.81, p<0.01$ ). Sequential Organ Failure Assessment (SOFA) score was correlated with L-carnitine reduction but without a significant cutoff value. Patients treated with CRRT demonstrated elevated A/F Carnitine ratio $(p<0.05)$, possibly due to insufficient elimination or impaired metabolism of carnitine.

Conclusions: Less than one fourth of critically ill patients had carnitine deficiency at ICU admission, while low BMI and high SOFA scores were identified as potential risk factors for reduction of L-carnitine. Patients treated with CRRT presented signs of impaired carnitine metabolism. Further studies to investigate the potential benefits of $\mathrm{L}$-carnitine supplementation may be warranted in these patients.

Trial registration: UMIN000013352. Registered 6 March 2014.

Keywords: Carnitine, Nutritional support, Critical care, Renal replacement therapy

\section{Background}

L-carnitine is an essential compound for energy utilization through lipid metabolism in the skeletal muscle [1]. It is mainly supplied through the regular diet, especially from animal meat and dairy products. It can also be synthesized endogenously from lysine and methionine in the liver and the kidneys. The endogenous composition of L-carnitine comprises of free carnitine and acylcarnitines (L-carnitine in the form of esters such as acetyl L-carnitine) [2, 3]. Carnitine deficiency can

\footnotetext{
* Correspondence: t_oshima@chiba-u.jp; oshima0528@gmail.com 'Department of Emergency and Critical Care Medicine, Chiba University Graduate School of Medicine, Chiba University Hospital, 1-8-1 Inohana Chuo-ku, Chiba, Chiba, Japan

Full list of author information is available at the end of the article
}

occur as a primary disorder due to the deficiency of $\mathrm{L}$-carnitine cellular transporters, but it is more prevalent as secondary deficiency as a result of conditions such as insufficient intake, medical treatments promoting excretion, and excessive elimination by hemodialysis [2].

Critically ill patients are usually nourished artificially by enteral or parenteral feeding, which often leads to the lack of L-carnitine intake. The patients are also frequently treated with continuous renal replacement therapy (CRRT) that may cause L-carnitine depletion, in a similar way to hemodialysis. Lack of L-carnitine impairs the utilization of fatty acid as energy source and leads to muscle weakness and muscle loss due to catabolism, which leads to deterioration of patients' physical function. 
It is also suggested to lead to anemia and immune dysfunction [1, 4], leading to infectious complications.

While the prevalence of carnitine deficiency and the effect of supplementation have been studied previously in postoperative patients [5, 6], L-carnitine concentrations of patients admitted to the intensive care unit (ICU) for unscheduled treatments have not been well described. However, these patients are more likely to be treated longer [7] with higher risks of organ failure treatment [7]. Thus, these patients are more likely to develop carnitine depletion and possibly benefit from supplementation. The present study was conducted to define the prevalence of carnitine deficiency and risk factors of L-carnitine depletion in adult critically ill patients admitted to the ICU for unscheduled treatment and to identify the potential indications for supplementation of carnitine. Further analysis was conducted to determine the indication of carnitine supplementation in subgroup of patients who underwent CRRT [8].

\section{Methods}

\section{Study design}

A prospective observational study to analyze the total and free L-carnitine concentrations at ICU admission and during the hospital stay was conducted on adult critically ill patients. The primary objective was to define the prevalence of carnitine deficiency at the time of ICU admission. The secondary objectives were to define patient characteristics at ICU admission that indicate risks for future carnitine depletion and to define ICU treatments that influence L-carnitine concentrations. Patients were treated according to local treatment protocols and were not supplemented with L-carnitine as a part of their treatments. All patient samples were collected strictly from residual serum samples from routine blood analysis at ICU admission and during the hospital stay.

\section{Patient selection}

Adult patients admitted to the ICU of Chiba University Hospital, for unscheduled treatment from February 1, 2014, to March 30, 2014, were enrolled. Patients were excluded if they were under the age of 18 years, were admitted to the ICU for post elective surgery care, readmitted to the ICU, or were not expected to survive for more than $24 \mathrm{~h}$ at the time of screening for eligibility.

\section{L-carnitine analysis}

Residual serum samples were collected for L-carnitine analyses. Initial samples were collected from the routine blood evaluation at the time of ICU admission and followed every 7 days during the hospitalization for a maximum of 28 days. As sample collection relied on residual serum, follow-up samples collected within 1 day before or after the scheduled follow-up date were considered valid for evaluation.

All blood samples were centrifuged immediately after sampling at the central clinical laboratory of Chiba University Hospital and stored in serum form at $4{ }^{\circ} \mathrm{C}$ for no longer than $72 \mathrm{~h}$. Samples were then collected and stored in the $-80{ }^{\circ} \mathrm{C}$ freezer until the analysis. L-carnitine concentrations were analyzed as total carnitine concentration (t-Carnitine) and free carnitine concentration (f-Carnitine) for all obtained samples. Acylcarnitine concentration was calculated as the difference of $\mathrm{t}$-Carnitine and $\mathrm{f}$-Carnitine concentrations (Acylcarnitine $=\mathrm{t}$ Carnitine - $\mathrm{f}$-Carnitine). $\mathrm{t}$-Carnitine and $\mathrm{f}$-Carnitine analyses were conducted by the enzyme cycling method (KAINOS laboratories Inc., Tokyo, Japan) [9] which uses carnitine dehydrogenase as the primary enzyme and thionicotinamide-adenine dinucleotide (thio-NAD+) and nicotinamide adenine dinucleotide (NADH) as the coenzymes. All samples were analyzed within 60 days from the sampling date. Preliminary tests were conducted to verify this procedure, which revealed no significant change in the measured results after 7-day storage at $4^{\circ}$ $\mathrm{C}$ or after 60 -day storage at $-80{ }^{\circ} \mathrm{C}$, compared to immediate analysis after sample processing (data not shown). The lower and the upper reference values of $\mathrm{f}$-Carnitine are 36 and $75 \mathrm{nmol} / \mathrm{mL}$, respectively, according to the specification by the manufacturer.

\section{Data collection}

Patient characteristics consisting of age, height, weight, body mass index (BMI), Acute Physiology and Chronic Health Evaluation (APACHE) II score, Sequential Organ Failure Assessment (SOFA) score, origin of admission, and underlying medical conditions were recorded for evaluation of relative factors for the L-carnitine concentration on admission to the ICU. Post-ICU admission treatments such as antibiotic administration, nutrition, and CRRT were recorded for the assessment of factors that may relate to the change in L-carnitine concentrations after ICU admission.

Clinical outcomes were assessed as mortality during the ICU stay (ICU mortality), during the hospital stay (hospital mortality), and at 180 days after ICU admission (180 -day mortality).

\section{Data analysis}

For the primary analysis, patients were classified into two groups according to the L-carnitine concentration at ICU admission: low carnitine group, patients with $\mathrm{f}$-Carnitine lower than the lower reference value $(36 \mathrm{nmol} /$ $\mathrm{mL}$ ), and normal carnitine group, patients with fCarnitine higher than the lower reference values. The patient background characteristics at the time of admission were also compared between the two groups to 
identify risk factors for carnitine depletion at the time of ICU admission.

For the secondary analyses, patients were classified into two groups according to the changes in L-carnitine concentrations from day 0 to day 14 of the hospital stay: carnitine reduction group (f-Carnitine at day $0>\mathrm{f}$-Carnitine at day 14) or carnitine elevation group (f-Carnitine at day $0 \leq \mathrm{f}$-Carnitine at day 14). Factors associated with the reduction or the elevation of L-carnitine concentrations were investigated by comparing the patient characteristics and treatments conducted after ICU admission.

Calculated acylcarnitine concentrations were studied as ratio to $\mathrm{f}$-Carnitine (A/F Carnitine ratio) to evaluate the need for L-carnitine supplementation in patients treated with continuous renal replacement therapy for acute kidney injury (AKI).

Data are presented as mean \pm standard deviation (SD), median and quartile, or absolute numbers and percentages as appropriate. We tested for differences between the two groups using an unpaired $t$ test for continuous data and Fisher's exact test for categorical data. Receiver operating characteristics (ROC) curve analysis was conducted for potential risk factors using f-Carnitine reduction at day 14 as the dependent variable. The cutoff value was determined by Youden-Index. Statistical analyses were performed using the GraphPad Prism 6 (GraphPad Software, San Diego, CA, USA).

\section{Results}

\section{Patient selection}

During the study period, 338 patients were admitted to the ICU. Sixty-four patients were enrolled in the study after excluding 224 post surgery patients, two patients under the age of 18 , six patients who were readmitted to the ICU, and five patients who were expected to die within $24 \mathrm{~h}$, by the exclusion criteria. Also, 37 patients were lost for enrollment due to other reasons such as insufficient residual serum samples and admission during holidays when the initial sample processing was not possible (Additional file 1: Figure S1).

\section{Primary outcome}

The median $\mathrm{t}$-Carnitine and $\mathrm{f}$-Carnitine at the time of admission were $64.2(50.5-102.3) \mathrm{nmol} / \mathrm{mL}$ and 50.5 (36.8-80.3) $\mathrm{nmol} / \mathrm{mL}$, respectively. Carnitine deficiency defined as $\mathrm{f}$-Carnitine $<36 \mathrm{nmol} / \mathrm{mL}$ was observed in 15 (23.4\%) patients (Table 1). No significant differences were found between the low carnitine group and the normal carnitine group age, sex, BMI, comorbidities, severity of the critical illness, or admission diagnoses (Table 1).

\section{Secondary outcomes}

\section{Transition of carnitine concentrations}

The median t-Carnitine and f-Carnitine at day 14, 71.7 (55.9-83.1) and 59.8 (46.0-69.1), respectively, were within the upper and lower reference values (Additional files 2 and 3).

\section{Factors related to the reduction or the elevation of $L-$ carnitine concentrations}

Patients were divided into carnitine elevation group $(n=14,48.3 \%)$ and carnitine reduction group $(n=15$, $51.7 \%)$ according to the 14-day transition of f-Carnitine (Table 2). The 14-day transitions of f-Carnitine concentrations in the two groups are shown in Table 2 and Additional file 3: Figure S3 in the supplementary appendix. BMI was significantly lower in the carnitine reduction group compared with the carnitine elevation group (19.4 \pm 2.9 and $22.4 \pm 2.8$, respectively, $p=0.01$ ). No significant differences were found in the patient severity at ICU admission (SOFA score and APACHE II score). There were no significant differences in the rates of ICU treatments such as administration of artificial nutrition; CRRT for AKI was also not statistically significant (Table 2).

The association between the clinical factors and the degree of change in $\mathrm{f}$-Carnitine from day 0 to day 14 $(\Delta \mathrm{f}$-Carnitine) were further analyzed (Table 3$)$. There was a significant negative correlation between the BMI and the $\Delta \mathrm{f}$-Carnitine $(p=0.01)$. ROC curve analysis revealed a significant cutoff value of 19.5 (area under the curve $($ AUC $)=0.81, p<0.01)($ Fig. 1a).

Significantly positive correlation was observed between the SOFA score on ICU admission and the $\triangle \mathrm{f}$-Carnitine $(p=0.012)$ (Additional file 4). However, a significant cutoff value was not defined by the ROC curve analysis (tentative cutoff value 8.5; AUC =0.57, $p=0.5$ ) (Fig. 1b).

\section{Contribution of CRRT on the A/F Carnitine ratio}

Patients treated with CRRT were compared with patients without CRRT. Although a significant difference of $\mathrm{f}$-Carnitine concentrations was not found among patients with CRRT and without CRRT at day 14, A/F Carnitine ratio at day 14 was higher in CRRT patients compared with the patients without CRRT $(p=0.012$, Fig. 2).

\section{Discussion}

In the present study, carnitine deficiency was observed in $23.4 \%$ of the critically ill patients admitted to the ICU for unexpected treatments. BMI lower than 19.5 was found to be a significant risk factor for L-carnitine reduction. SOFA score at the time of ICU admission was correlated with the subsequent decrease in L-carnitine concentrations, although a significant cutoff value was not defined. 
Table 1 Primary outcome and patient characteristic at ICU admission

\begin{tabular}{|c|c|c|c|c|}
\hline & All patients & f-Carnitine ${ }^{\ddagger}<36$ & f-Carnitine $e^{\ddagger}>36$ & $p$ value \\
\hline Patients (\%) & $64(100.0)$ & $15(23.4)$ & 49 (76.6) & \\
\hline f-Carnitine ${ }^{\ddagger}$ & $50.5(36.8-80.3)$ & $30.0(25.5-34.2)$ & $57.9(46.0-100.3)$ & $<0.01$ \\
\hline Age (year) & $62.8 \pm 17.4$ & $60.1 \pm 17.3$ & $63.6 \pm 17.5$ & 0.49 \\
\hline Male (\%) & $41(64.0)$ & $7(46.6)$ & $34(69.3)$ & 0.13 \\
\hline Weight (kg) & $57.4 \pm 11.7$ & $58.7 \pm 9.6$ & $56.7 \pm 12.3$ & 0.56 \\
\hline Body mass index & $21.6 \pm 3.8$ & $23.3 \pm 2.9$ & $21.1 \pm 3.9$ & 0.06 \\
\hline \multicolumn{5}{|l|}{ Comorbidity } \\
\hline Diabetes mellitus & $10(15.6)$ & $3(20.0)$ & $7(14.2)$ & 0.68 \\
\hline Hyperlipidemia & $3(4.6)$ & $0(0)$ & $3(6.1)$ & 1.0 \\
\hline Cirrhosis & $4(6.2)$ & $1(6.6)$ & $3(6.1)$ & 1.0 \\
\hline Chronic kidney disease & $5(7.8)$ & $2(13.3)$ & $3(6.1)$ & 0.3 \\
\hline Hemodialysis & $4(6.2)$ & $2(13.3)$ & $2(4.0)$ & 0.23 \\
\hline APACHE $\|^{\natural}$ score & $26.9 \pm 10.4$ & $25.5 \pm 10.8$ & $27.4 \pm 10.3$ & 0.56 \\
\hline SOFA score $^{\S}$ & $8.1 \pm 4.8$ & $6.1 \pm 3.9$ & $8.8 \pm 5.0$ & 0.06 \\
\hline \multicolumn{5}{|l|}{ Diagnosis, no. (\%) } \\
\hline Sepsis & $18(28.1)$ & $2(13.3)$ & $16(32.6)$ & 0.19 \\
\hline Gastrointestinal disorder & $13(20.3)$ & $5(33.3)$ & $8(16.3)$ & 0.16 \\
\hline Neurological disorder & $9(14.0)$ & $3(20)$ & $6(12.2)$ & 0.42 \\
\hline Trauma & $10(15.6)$ & $2(13.3)$ & $8(16.3)$ & 1.0 \\
\hline Patient origin & & & & 0.08 \\
\hline Emergency department & $22(34.3)$ & $8(53.3)$ & $14(28.5)$ & \\
\hline General ward & $17(26.5)$ & $1(6.6)$ & $16(32.6)$ & \\
\hline Other hospital & $25(39.0)$ & $6(40.0)$ & 19 (38.7) & \\
\hline Fasting duration (days) & $1.1 \pm 0.9$ & $1.0 \pm 0.6$ & $1.2 \pm 1.0$ & 0.45 \\
\hline Artificial nutrition & $5(7.8)$ & $1(6.6)$ & $4(8.1)$ & 1.0 \\
\hline Valproic acid treatment & $2(3.1)$ & 0 & $2(4.0)$ & 1.0 \\
\hline Alb (g/dL) & $2.9 \pm 0.8$ & $3.1 \pm 0.7$ & $2.8 \pm 0.8$ & 0.21 \\
\hline $\mathrm{HbA1c}(\%)$ & $6.4 \pm 2.5$ & $6.1 \pm 2.3$ & $6.4 \pm 2.6$ & 0.73 \\
\hline
\end{tabular}

Data are presented as mean \pm standard deviation, median (quartile), or absolute numbers and percentages (\%) as appropriate

${ }^{\mp}$ Free carnitine

"Acute Physiology and Chronic Health Evaluation II

${ }^{5}$ Sequential Organ Failure Assessment

\section{Carnitine deficiency in ICU patients}

This study is the first epidemiological study to prospectively investigate the L-carnitine concentrations in critically ill patients who were admitted to the ICU for unscheduled treatment. Previous studies in postoperative patients have concluded that carnitine deficiency was rarely observed [5] and that the benefit of supplementation was limited $[6,10,11]$. Recent progress in ICU treatments including organ support therapies has enabled critically ill patients of various etiologies and severity to survive the critical illness and to endure longterm intensive treatments. However, little has been studied about carnitine concentrations and its roles in the current-day critically ill patients. Recent studies regarding carnitine concentrations have focused on non-critically ill patients $[4,12,13]$ or critically ill patients already presenting symptoms of carnitine depletion $[1,14]$.

\section{Indication for L-carnitine measurement and supplementation}

There were no significant differences in the clinical outcomes such as length of ICU stay and mortality between patients with or without carnitine deficiency at the time of ICU admission (Additional file 5: Table S1). This observation suggests that carnitine deficiency at ICU admission does not immediately indicate the need for carnitine supplementation. However, long-term multidisciplinary treatments provided for current critically ill patients while lacking sufficient supplementation could increase the chance of carnitine depletion in the later 
Table 2 Secondary outcomes

\begin{tabular}{|c|c|c|c|}
\hline & Carnitine elevation group $(n=14)$ & Carnitine reduction group $(n=15)$ & $p$ value \\
\hline $\begin{array}{l}\text { f-Carnitine } e^{\ddagger} \text { change from day } 0 \text { to } \\
\text { day } 14\left(\Delta \text {-Carnitine } e^{\ddagger}\right)\end{array}$ & $12.2(4.7 \sim 26.9)$ & $-22.3(-34.8 \sim-4.4)$ & $<0.01$ \\
\hline Age & $64.2 \pm 17.0$ & $61.8 \pm 11.5$ & 0.66 \\
\hline Body mass index & $22.4 \pm 2.8$ & $19.4 \pm 2.9$ & 0.01 \\
\hline ICU duration & $7.5(3.0 \sim 13.7)$ & $12.0(5.0 \sim 21.0)$ & 0.14 \\
\hline Sepsis during ICU stay & $6(40.0)$ & $6(42.8)$ & 0.87 \\
\hline APACHE $\|^{\natural}$ score on admission & $26.6 \pm 8.7$ & $29.4 \pm 8.8$ & 0.40 \\
\hline SOFA $^{\S}$ score on admission & $8.0 \pm 3.6$ & $9.0 \pm 4.6$ & 0.53 \\
\hline Mechanical ventilation during ICU stay & $0.0(0.0 \sim 2.0)$ & $4.0(0.0 \sim 8.0)$ & 0.08 \\
\hline Artificial nutrition at day 14 & $7(42.8)$ & $8(53.3)$ & 1.0 \\
\hline Parenteral nutrition at day 14 & $3(21.4)$ & $0(0)$ & 0.1 \\
\hline Enteral nutrition at day 14 & $4(28.5)$ & $8(53.3)$ & 0.26 \\
\hline Calorie intake per weight at day 14 & $26.4 \pm 9.2$ & $28.8 \pm 8.4$ & 0.46 \\
\hline Renal replacement therapy during ICU stay & $6(42.8)$ & $9(60.0)$ & 0.46 \\
\hline
\end{tabular}

Data are presented as mean \pm standard deviation, median (quartile), or absolute numbers and percentages (\%) as appropriate

${ }^{\ddagger}$ Free carnitine

"Acute Physiology and Chronic Health Evaluation II

${ }^{\S}$ Sequential Organ Failure Assessment

phase of the ICU stay. Although the median L-carnitine concentration for the ICU patients did not fall under the reference range during the 14-day study period, L-carnitine concentrations progressively declined in $51.7 \%$ of the patients. Medical treatments such as pivampicillin, valproic acid, and verapamil administration can lead to L-carnitine deficiency by either inhibiting cellular transporters or promoting L-carnitine excretion. Extended artificial nutrition is also a frequent cause of the depletion due to the lack of L-carnitine content in enteral or intravenous nutrition formula. L-carnitine is sufficiently pooled endogenously; thus, carnitine depletion usually occurs after more than 14 days of interrupted carnitine supply [14]. Evaluation over longer stay is likely to reveal further decrease of L-carnitine concentration, as

Table 3 Correlation between clinical factors and f-Carnitine ${ }^{\ddagger}$ change from day 0 to day $14\left(\Delta\right.$-Carnitine $\left.{ }^{\ddagger}\right)$

\begin{tabular}{|c|c|c|}
\hline & $r^{\dagger}$ & $p$ value \\
\hline Body mass index & -0.47 & 0.01 \\
\hline SOFA $A^{\S}$ score on admission & 0.46 & 0.01 \\
\hline APACHE $\|^{\natural}$ score on admission & 0.32 & 0.1 \\
\hline Length of renal replacement therapy & 0.35 & 0.06 \\
\hline Mechanical ventilation during ICU stay & 0.27 & 0.14 \\
\hline Calorie intake per weight at day 14 & -0.01 & 0.95 \\
\hline Serum Alb ( $\mathrm{g} / \mathrm{dL}$ ) on admission & 0.16 & 0.40 \\
\hline ICU length of stay & 0.25 & 0.17 \\
\hline
\end{tabular}

FFree carnitine

"Acute Physiology and Chronic Health Evaluation II

${ }^{5}$ Sequential Organ Failure Assessment

${ }^{+}$Correlation analyzed with Spearman coefficient demonstrated in some case reports of carnitine depletion $[1,14]$.

The relationship between BMI and decreased L-carnitine concentrations may be related to the fact that L-carnitine is mainly stored in the skeletal muscles, where it facilitates medium- and long-chain fatty acid transport from the cytosol into the mitochondria for $\beta$-oxidation and energy generation $[1,4]$. Since muscle mass is frequently lost during the early phase of the critical illness due to catabolism [15], patients with smaller muscle mass, possibly reflected by low BMI, are likely to suffer loss of relatively greater portion of their carnitine pool, leading to decreased L-carnitine concentrations. However, more specific methods to evaluate muscle mass such as the body composition $[16,17]$ analysis or anatomical evaluations $[18,19]$ are needed to confirm this hypothesis. It should also be noted that the study population was limited to patients with relatively low BMI (mean \pm SD $21.6 \pm 3.8$ ).

The mechanism behind carnitine reduction in patients with higher SOFA scores is not well defined. One explanation may be the metabolic load on mitochondrial function during severe illness [20]. One randomized controlled trial studied the effect of carnitine infusion in vasopressor-dependent septic shock patients based on this hypothesis [20]. Although carnitine concentrations were not measured in this study, early infusion of L-carnitine was related to reduced 28-day mortality without harmful effects, suggesting a potential benefit of L-carnitine infusion in this patient population. Despite the uncertainty of the mechanism, these findings warrant further investigation of $\mathrm{L}$-carnitine concentrations in 

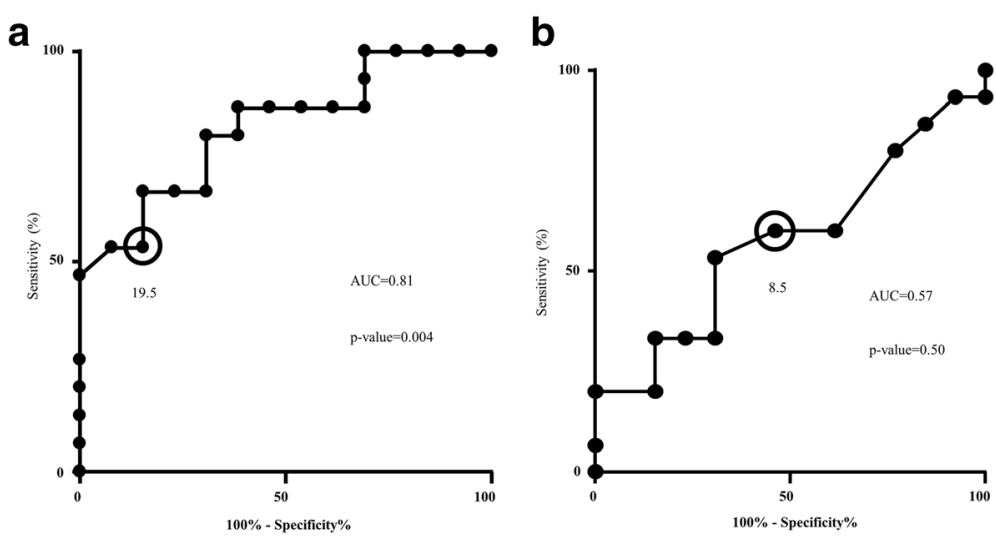

Fig. 1 ROC curve analysis for BMI and SOFA score associated with f-Carnitine reduction at day 14. Each plot depicts a relationship between sensitivity on the $x$-axis and 100-specificity on the $y$-axis. The cutoff value of BMI (a) was defined as 19.5, with the ROC curve depicting area under the curve (AUC) of 0.81 (95\% confidence interval 0.66-0.97). A significant cutoff value was not defined for SOFA score (b) (tentative cutoff value 8.5; AUC $=0.57,95 \%$ confidence interval 0.35-0.78)

relationship to the benefits of carnitine supplementation in patients with organ dysfunctions.

\section{Patients treated with CRRT}

Indication for supplementation of carnitine can also be determined by focusing on the accumulation of acylcarnitine by its ratio to free carnitine (A/F Carnitine ratio), as implemented in hemodialysis patients [8]. The accumulation of acylcarnitine in the blood indicates impaired mitochondrial metabolism, insufficient elimination by the kidney, or impaired transport of acylcarnitine due to the lack of L-carnitine supply and can lead to complications such as liver mitochondrial dysfunction [2]. The administration of L-carnitine in hemodialysis patients has been suggested to help the redistribution of acylcarnitine to eliminate potentially toxic acylcarnitines through hemodialysis [8]. The subgroup of patients

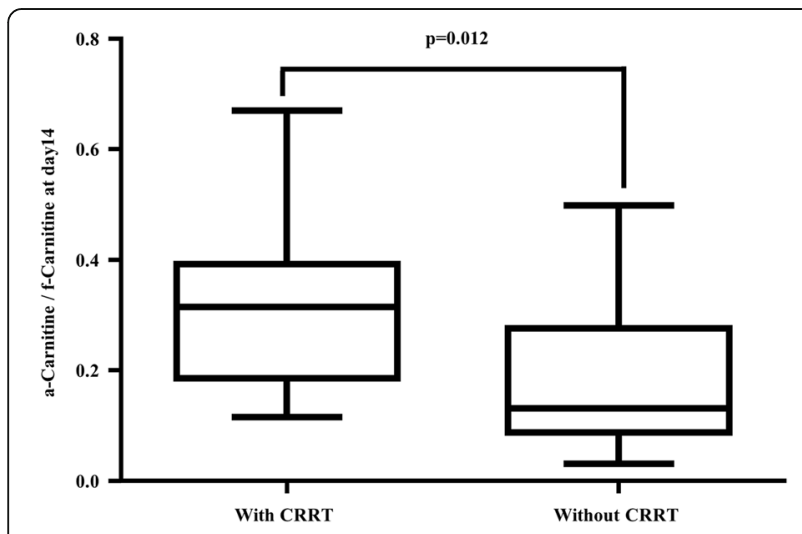

Fig. 2 Comparison of a-Carnitine/f-Carnitine ratio between the patients treated with or without CRRT. The box depicts the interquartile range and the line within the box indicating the median, respectively. The error bars indicate the 25th and 75th percentile range. The a-Carnitine/f-Carnitine ratio at day 14 was higher in patients with CRRT $(p=0.01)$ treated with CRRT presented elevated A/F Carnitine ratio, a finding consistent with hemodialysis patients [13]. The difference in the efficacy of RRT implicates a disparate mechanism affecting A/F Carnitine ratio in patients with CRRT compared to hemodialysis patients. In critically ill patients usually treated with CRRT, the relationship between mitochondrial dysfunction and severity has been shown in the previous reports $[21,22]$. We speculate that not only insufficient elimination by kidneys but also impaired mitochondrial metabolism due to critical illness lead to accumulation of acylcarnitine. Although dialysis efficiency of CRRT is different from that of hemodialysis, carnitine supplementation in ICU patients treated with CRRT may help to avoid symptoms similar to dialysis-related complications such as anemia and muscle weakness [13].

\section{Limitations}

This study has some limitations, mainly related to the study design. The results obtained from limited patient enrollment in a single-center observation require external validation of the results. Valid patient follow-up was possible for only 14 days, while carnitine depletion is more likely to occur in long-term treatments [14]. Although we were able to identify the trend of carnitine reduction in patients with lower BMI and higher SOFA scores, the transition of carnitine concentrations were within the reference ranges. Longer follow-up in a larger patient population may have enabled more specific analyses, including the possibility of identifying other risk factors. The study design to use BMI as the only parameter for the estimation of endogenous carnitine supply is another limitation, as more specific methods are recommended to determine muscle mass $[18,19]$. On the other hand, the contribution of carnitine metabolism on anemia and decreased cardiac performance, which is 
known to be significant for hemodialysis patients [23], should be investigated in critically ill patients. Despite these limitations, the present study attempted to collect the epidemiological data on carnitine concentrations in modern critically ill patients and to identify the potential focuses for future studies.

\section{Conclusions}

Carnitine deficiency was observed in less than one fourth of critically ill patients at ICU admission. Low BMI $(<19.5)$ and high SOFA scores at ICU admission were identified as potential risk factors for carnitine depletion during extended ICU stay. Patients treated with CRRT presented signs of impaired carnitine metabolism. Further studies to investigate the potential benefits of carnitine supplementation may be warranted for these patients.

\section{Additional files}

Additional file 1: Figure S1. Flowchart of study enrollment. (TIFF $117 \mathrm{~kb}$ ) Additional file 2: Figure S2. Distribution of carnitine concentrations on ICU admission. (a) The distribution of t-Carnitine on ICU admission is shown in the histogram. The mean t-Carnitine was $94.9 \pm 79.6 \mathrm{nmol} / \mathrm{mL}$, and the median t-Carnitine was $64.2(50.5-102.3) \mathrm{nmol} / \mathrm{mL}$. (b) The distribution of $\mathrm{f}$-Carnitine on ICU admission is shown in the histogram. The mean f-Carnitine was $68.6 \pm 51.2 \mathrm{nmol} / \mathrm{mL}$, and the median f-Carnitine was 50.5 (36.880.3) $\mathrm{nmol} / \mathrm{mL}$. (ZIP $160 \mathrm{~kb})$

Additional file 3: Figure S3. Comparison of f-Carnitine transition among the patients with f-Carnitine increased and decreased. The transition of f-Carnitine area for 14 days plotted at each sampling point. The round and square signs within the figure indicate the medians for $\mathrm{f}$-Carnitine, and the error bars indicate 25th and 75th percentile range. The dotted lines indicate the upper and lower reference values for f-Carnitine. The patients whose data are lacking at day 7 or day 14 were excluded from this analysis. (TIFF $123 \mathrm{~kb}$ )

Additional file 4: Figure S4. Correlation of BMI and SOFA score with the change in $\mathrm{f}$-Carnitine from day 0 to day 14. (a) Each plot depicts a relationship between the BMI on the $x$-axis, and the change of $f$-Carnitine from day 0 to day 14 ( $\Delta \mathrm{f}$-Carnitine) on the $y$-axis. There was a significant negative correlation between the BMl and the $\Delta f$-Carnitine $(r=-0.47, p=0.01)$, which suggests an association between lower BMl and larger decrease of f-Carnitine. (b) Each plot depicts a relationship between the SOFA score at the time of ICU admission on the $x$-axis, and the change of f-Carnitine from day 0 to day 14 ( $\Delta$ f-Carnitine) on the $y$-axis. There was a significant correlation between the SOFA score on ICU admission and the $\Delta f$-Carnitine $(r=0.46, p=0.01)$, which suggests an association between high SOFA score and larger decrease of f-Carnitine. (ZIP $188 \mathrm{~kb}$ )

Additional file 5: Table S1. Clinical outcomes according to f-carnitine levels at ICU admission ${ }^{\mathrm{a}}$. (DOCX $\left.21 \mathrm{~kb}\right)$

\section{Abbreviations}

AKI: Acute kidney injury; APACHE: Acute Physiology and Chronic Health Evaluation; BMI: Body mass index; CRRT: Continuous renal replacement therapy; f-Carnitine: Free carnitine concentration; ICU: Intensive care unit; NADH: Nicotinamide adenine dinucleotide; ROC: Receiver operating characteristics; SD: Standard deviation; SOFA: Sequential Organ Failure Assessment; t-Carnitine: Total carnitine concentration; thio-NAD

${ }^{+}$: Thionicotinamide-adenine dinucleotide

\section{Acknowledgements}

Not applicable

\section{Funding}

This research did not receive any specific grant from funding agencies in the public, commercial, or not-for-profit sectors.
Test reagents for $\mathrm{t}$-Carnitine and f-Carnitine analyses were provided by KAlNOS Laboratories Inc. (Tokyo, Japan) who had no involvement in the designing of the study, interpretation of the data, or the drafting of the manuscript. Carnitine analyses were conducted by the courtesy of the Central Clinical Testing Laboratory at Chiba University Hospital.

\section{Availability of data and materials}

The datasets used and analyzed during the current study are available from the corresponding author on reasonable request.

\section{Authors' contributions}

TO planned and conducted the study, analyzed the data, and drafted the manuscript. TOs planned and conducted the study and drafted the manuscript. $\mathrm{NH}$ made suggestions for the interpretation of the data and drafted the manuscript. AT recruited patients, collected patient samples, and critically revised the manuscript. SH and TY collected the samples, conducted the sample analysis, and critically revised the manuscript. SO supervised the study and drafted the manuscript. All authors gave the final approval for the final version of the manuscript prior to submission.

\section{Ethics approval and consent to participate}

The study protocol was approved at the ethical committee at Chiba University Graduate School of Medicine, as an epidemiological study conducted on residual patient blood samples. Written consent for enrollment was obtained from patients and/or their legal representatives as an agreement at ICU admission for the extended use of residual serum samples obtained for usual clinical practice. Patients and/or their legal representatives were informed about the details of the study by a documented explanation form and were given the right to withdraw consent after enrollment.

\section{Competing interests}

The authors declare that they have no competing interests.

\section{Publisher's Note}

Springer Nature remains neutral with regard to jurisdictional claims in published maps and institutional affiliations.

\section{Author details}

${ }^{1}$ Department of Emergency and Critical Care Medicine, Chiba University Graduate School of Medicine, Chiba University Hospital, 1-8-1 Inohana Chuo-ku, Chiba, Chiba, Japan. ${ }^{2}$ Clinical Laboratory, Chiba University Hospital, Chiba, Japan.

Received: 10 July 2017 Accepted: 22 February 2018

Published online: 11 April 2018

\section{References}

1. Hatamkhani S, Karimzadeh I, Elyasi S, Farsaie S, Khalili H. Carnitine and sepsis: a review of an old clinical dilemma. J Pharm Pharm Sci. 2013;16:414-23.

2. Reuter SE, Evans AM. Carnitine and acylcarnitines: pharmacokinetic, pharmacological and clinical aspects. Clin Pharmacokinet. 2012;51:553-72.

3. Cao Y, Wang YX, Liu CJ, Wang LX, Han ZW, Wang CB. Comparison of pharmacokinetics of $\mathrm{L}$-carnitine, acetyl-L-carnitine and propionyl-L-carnitine after single oral administration of L-carnitine in healthy volunteers. Clin Invest Med. 2009;32:E13-9.

4. Calo LA, Vertolli U, Davis PA, Savica V. L carnitine in hemodialysis patients. Hemodial Int. 2012;16:428-34.

5. Wennberg A, Hyltander A, Sjoberg A, Arfvidsson B, Sandstrom R, Wickstrom I, Lundholm K. Prevalence of carnitine depletion in critically ill patients with undernutrition. Metabolism. 1992;41:165-71.

6. Pichard C, Roulet M, Schutz Y, Rossle C, Chiolero R, Temler E, Schindler C, Zurlo F, Furst $P$, Jequier $E$. Clinical relevance of $L$-carnitine-supplemented total parenteral nutrition in postoperative trauma. Metabolic effects of continuous or acute carnitine administration with special reference to fat oxidation and nitrogen utilization. Am J Clin Nutr. 1989;49:283-9.

7. Ostermann ME, Chang RW. Prognosis of acute renal failure: an evaluation of proposed consensus criteria. Intensive Care Med. 2005;31:250-6.

8. Vernez L, Dickenmann M, Steiger J, Wenk M, Krahenbuhl S. Effect of Lcarnitine on the kinetics of carnitine, acylcarnitines and butyrobetaine in long-term haemodialysis. Nephrol Dial Transplant. 2006;21:450-8. 
9. Takahashi M, Ueda S, Misaki H, Sugiyama N, Matsumoto K, Matsuo N, Murao S. Carnitine determination by an enzymatic cycling method with carnitine dehydrogenase. Clin Chem. 1994;40:817-21.

10. Roulet M, Pichard C, Rossle C, Bretenstein E, Schutz Y, Chiolero R, Furst P, Jequier $E$. Adverse effects of high dose carnitine supplementation of total parenteral nutrition on protein and fat oxidation in the critically ill. Clin Nutr. 1989:8:83-7.

11. Sandstedt S, Cederblad G, Lindholm M, Larsson J. The effect of carnitine supplemented total parenteral nutrition on lipid, energy and nitrogen metabolism in severely ill patients. Clin Nutr. 1991;10:97-104.

12. Ringseis R, Keller J, Eder K. Role of carnitine in the regulation of glucose homeostasis and insulin sensitivity: evidence from in vivo and in vitro studies with carnitine supplementation and carnitine deficiency. Eur J Nutr. 2012;51:1-18.

13. Evans AM, Faull RJ, Nation RL, Prasad S, Elias T, Reuter SE, Fornasini G Impact of hemodialysis on endogenous plasma and muscle carnitine levels in patients with end-stage renal disease. Kidney Int. 2004;66:1527-34.

14. Bonafe L, Berger MM, Que YA, Mechanick Jl. Carnitine deficiency in chronic critical illness. Curr Opin Clin Nutr Metab Care. 2014;17:200-9.

15. Puthucheary ZA, Rawal J, McPhail M, Connolly B, Ratnayake G, Chan P, Hopkinson NS, Phadke R, Dew T, Sidhu PS, et al. Acute skeletal muscle wasting in critical illness. JAMA. 2013:310:1591-600

16. Ismael S, Savalle M, Trivin C, Gillaizeau F, D'Auzac C, Faisy C. The consequences of sudden fluid shifts on body composition in critically ill patients. Crit Care. 2014;18:R49.

17. Faisy C, Rabbat A, Kouchakji B, Laaban JP. Bioelectrical impedance analysis in estimating nutritional status and outcome of patients with chronic obstructive pulmonary disease and acute respiratory failure. Intensive Care Med. 2000;26:518-25.

18. Mourtzakis M, Wischmeyer P. Bedside ultrasound measurement of skeletal muscle. Curr Opin Clin Nutr Metab Care. 2014;17:389-95.

19. Moisey LL, Mourtzakis M, Cotton BA, Premji T, Heyland DK, Wade CE, Bulger E, Kozar RA. Skeletal muscle predicts ventilator-free days, ICU-free days, and mortality in elderly ICU patients. Crit Care. 2013;17:R206.

20. Puskarich MA, Kline JA, Krabill V, Claremont $\mathrm{H}$, Jones AE. Preliminary safety and efficacy of $\mathrm{L}$-carnitine infusion for the treatment of vasopressordependent septic shock: a randomized control trial. JPEN J Parenter Enteral Nutr. 2014;38:736-43.

21. Brealey D, Brand M, Hargreaves I, Heales S, Land J, Smolenski R, Davies NA Cooper CE, Singer M. Association between mitochondrial dysfunction and severity and outcome of septic shock. Lancet. 2002;360:219-23.

22. Carre JE, Orban JC, Re L, Felsmann K, Iffert W, Bauer M, Suliman HB, Piantadosi CA, Mayhew TM, Breen P, et al. Survival in critical illness is associated with early activation of mitochondrial biogenesis. Am J Respir Crit Care Med. 2010;182:745-51.

23. Calvani M, Benatti P, Mancinelli A, D'Iddio S, Giordano V, Koverech A, Amato $A$, Brass EP. Carnitine replacement in end-stage renal disease and hemodialysis. Ann N Y Acad Sci. 2004;1033:52-66.

\section{Submit your next manuscript to BioMed Central and we will help you at every step:}

- We accept pre-submission inquiries

- Our selector tool helps you to find the most relevant journal

- We provide round the clock customer support

- Convenient online submission

- Thorough peer review

- Inclusion in PubMed and all major indexing services

- Maximum visibility for your research

Submit your manuscript at www.biomedcentral.com/submit

Biomed Central 